\title{
Influence of tensile cracking and of aggregate size on concrete permeability
}

\author{
Marta Choinska ${ }^{1,{ }^{*}, \text { Hayder Al-Khazraji }}{ }^{1,2}$, Nathan Benkemoun ${ }^{1}$, and Abdelhafid Khelidj ${ }^{1}$ \\ ${ }^{1}$ Institut de Recherche en Génie Civil et Mécanique GeM - UMR CNRS 6183, Université Bretagne \\ Loire, Université de Nantes, IUT Saint Nazaire, 58 rue Michel Ange, 44600 Saint-Nazaire, France \\ ${ }^{2}$ Missan University, Engineering College, Iraq
}

\begin{abstract}
The aim of this study is to investigate the interaction between crack opening (COD), aggregate size and gas transfer in concrete submitted to mechanical loading in the Brazilian splitting tensile test. The lab-made devices have been developed to investigate physical phenomena during loading and to provide data to validate a developed mesoscale hydromechanical model by the same authors, based upon a 3D lattice approach to represent the heterogeneity of the material [1]. Experimental studies has been carried out on five materials with different aggregate sizes. The results emphasize that permeability of mortar increases with cracking following a sigmoid law, with the most important kinetics due to passingthrough connected crack growth, after nonsymmetric one-face crack initiation. Furthermore, the obtained results highlight that permeability increase, due to aggregate size, may be separated from permeability increase due to tensile cracking: for all the five materials tested results fall on the same master sigmoid curve. This behaviour law represents a strong advantage for concrete modelling.
\end{abstract}

\section{Introduction}

Permeability is a parameter that may indirectly influence the durability of concrete structures by governing the penetration rate of aggressive agents responsible for degradation under a pressure gradient, or directly in the case of structures with tightness role. Hence, there is a clear motivation to investigate the variation of the permeability with cracking of concretelike materials during loading.

On the one hand, existing experimental data on tensile behavior provide some correlations between material damage and permeability, but they are in general limited by the residual permeability measurements performed after complete unloading [2-5]. Some studies [6-7] have been performed during loading but concern only water permeability, which remains delicate for interpretation as far as autogenous healing phenomena and water flow reduction are met [8-9]. Therefore, there is a clear need of data on intrinsic permeability evolution during tensile loading.

On the other hand, aggregate size should influence mechanical behaviour and permeability. Bisschop and van Mier [10] observed drying shrinkage microcracking increase

\footnotetext{
* Corresponding author: Marta.Choinska@univ-nantes.fr
} 
in cement-based composites with spherical glass aggregates size and with volume percentage increases. Grassl et al. [11] pointed out that the concrete permeability increases with the increasing of crack width and with the aggregate diameter. Grabiec et al. [12] highlighted the influence of the type and maximum aggregate size on some properties of high strength concrete. Fabien et al. [13] emphasized permeability increase with limestone aggregate size and compressive damage: higher aggregate specific surface of smaller aggregates impacts on higher cohesion and results in lower damage and lower permeability, independently of cement nature (CEM I or CEM III).

This work has therefore two main objectives: the first one concerns the evolution of permeability under tensile loading and the second one is focused on the effects of aggregate size on mechanical behavior and permeability. The authors use experimental methods to investigate these issues. The experimental results will be discussed in the light of comparison with numerical simulations based on a mesoscale model, developed by the same authors [1].

\section{Experimental program}

For the sake of simplicity, experimental study has been restricted to dry materials with mix proportions given in Tables 1 and 2 .

Table 1. Mortar constituents and mix proportions.

\begin{tabular}{|c|c|}
\hline Ingredient & $\begin{array}{c}\text { Quantity } \\
\left(\mathbf{k g} / \mathbf{m}^{\mathbf{3}}\right)\end{array}$ \\
\hline Cement (CEM I 52.5R PM-ES) & 451 \\
\hline Sand (alluvial rolled Quartz, Loire) 0-4mm & 1517 \\
\hline Water & 248 \\
\hline
\end{tabular}

Table 2. Microconcrete, concretes and macroconcrete constituents and mix proportions.

\begin{tabular}{|c|c|c|c|}
\hline \multirow{2}{*}{ Ingredient } & \multicolumn{3}{|c|}{ Quantity (kg/m³) } \\
\hline & Microconcrete & Concrete & Macroconcrete \\
\hline $\begin{array}{c}\text { Cement (CEM I 52.5R PM-ES or } \\
\text { CEM III/A 52.5 PM ES) }\end{array}$ & 350 & $\begin{array}{c}350 \text { or } \\
350 \\
\end{array}$ & 350 \\
\hline Sand (Limestone) $0-4 \mathrm{~mm}$ & \multicolumn{3}{|c|}{868} \\
\hline Medium aggregate (Limestone) $4-12 \mathrm{~mm}$ & 1009 & 432 & - \\
\hline Coarse aggregate (Limestone) 12-20mm & - & 561 & 981 \\
\hline Superplasticizer (solid content 20\%) & \multicolumn{3}{|c|}{1.71} \\
\hline Water & \multicolumn{3}{|c|}{168} \\
\hline
\end{tabular}

Material presented in Table 1 corresponds to a mortar, with water-to-cement ratio equal to 0.55 and aggregate volume fraction equal to $60 \%$.

All the materials presented in Table 2 have the same water-to-cement ratio, equal to 0.48 , as well as the same aggregate volume fraction, equal to $66 \%$ of the total volume. All the aggregates are crushed limestone. In order to investigate aggregate size effect on mechanical and transport properties, three types of formulations have been proposed: microconcrete, concrete and macroconrete. Microconcrete contains sand and medium size aggregates, two concretes contain sand, medium and coarse size aggregates and macroconcrete contains sand and coarse size aggregates only [13].

Cylindrical specimens (diameter of $110 \mathrm{~mm}$, length of $220 \mathrm{~mm}$ ) of mortar, microconcrete, two concretes and macroconcrete have been prepared from a single batch and cast in steel or PVC rigid moulds. $24 \mathrm{~h}$ after casting, they have been stored in a room maintained at $20^{\circ} \mathrm{C}$ with a $95 \%$ relative humidity, and then cured in water at $20^{\circ} \mathrm{C}$ for 4 weeks. After the storage 
in water, the cylinders have been cut using a diamond blade saw in order to obtain three "disc" specimens (diameter of $110 \mathrm{~mm}$, length of $47 \mathrm{~mm}$ ) within each cylinder. Mortar "disc" specimens have then been dried at $80^{\circ} \mathrm{C}$ down to constant mass, and then placed in a desiccator to keep their dry state. Microconcrete, concretes and macroconcrete "disc" specimens have been dried at $80^{\circ} \mathrm{C}$ over 2 weeks, then at $105^{\circ} \mathrm{C}$ down to constant mass.

\subsection{Tensile loading - gas permeability set-up}

For many years, our lab (GeM) has been working and developing coupled mechanical and permeability tests. After a compressive loading - gas permeability set-up [14], we propose a tensile loading - gas permeability set-up [15], based on indirect tension in the Brazilian splitting test (compression in the diameter plane). The principle of this test is to perform gas permeability measurements during loading, for different levels of controlled cracking in the pre-peak and post-peak phases, using a "disc" specimen loaded in the Brazilian splitting test. This test presents the advantage of forming a single crack, parallel to the loading direction $[2-5,7,16]$, where openings may be measured at one or two sides of a specimen, using f.ex. a COD sensor (crack-mouth opening displacement) as a feedback signal during the test [16]. Two devices, called a "simple" and a "double" one, have been developed in our lab and are presented in the next section.

\subsubsection{Developed experimental devices}

A simplified diagram of the "simple" loading-permeability device is shown in Fig. 1.

(a)

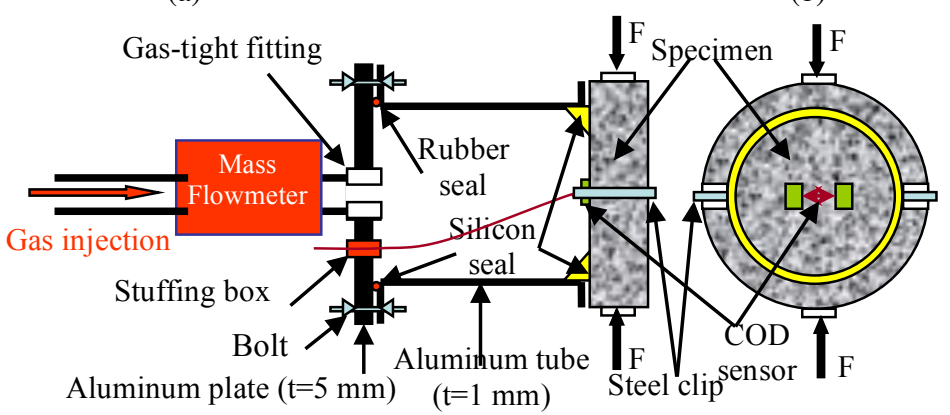

(c)

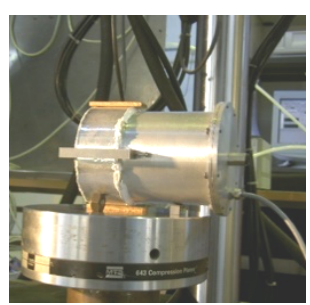

Fig. 1. Diagram of the "simple" experimental device to measure crack opening displacement (COD) and gas permeability during loading in the Brazilian splitting test: (a) side section view (b) front section view (c) overall view (GeM, Ecole Centrale de Nantes).

An inert gas (dry nitrogen) is injected into the cylindrical lightweight aluminium compartment, sealed to the specimen and maintained by two steel clips (see Fig. 1). The gas flow occurs in the direction perpendicular to the disc and thus parallel to the crack plane. In order to ensure a uniaxial gas flow and to prevent any parasitic gas leakage, the lateral face and the outer ring part of the up-stream side of a cylindrical specimen are protected by an adhesive aluminium film. The gas is injected at the up-stream side of a specimen at a pressure $\mathrm{Pi}$, equal here to $0.2 \mathrm{MPa}$ (absolute), the down-stream side being at atmospheric pressure $\left(\mathrm{P}_{\mathrm{atm}}\right)$. Injection pressure is applied and maintained until gas flow stabilization. This pressure is recorded during the test, as well as the flow rate, parallel to the recording of mechanical data (COD, axial force F, see Fig. 1). The relative pressure and mass flow rate are measured up-stream of the specimen by a thermal mass flowmeter which converts a mass flow rate to an equivalent volumetric gas flow rate at normalized conditions $\left(\mathrm{P}_{0}, \mathrm{~T}_{0}\right)$ [14]. Using Darcy's 
law, mass balance equation and the thermal mass flowmeters principle, an apparent permeability $\mathrm{k}\left(\mathrm{m}^{2}\right)$ may be calculated as follows (1):

$$
k\left(T_{a}\right)=\frac{2 Q_{i}\left(T_{0}, P_{0}\right) P_{0} \frac{T_{a}}{T_{0}} \mu\left(T_{a}\right) L}{S\left(P_{i}^{2}-P_{a t m}{ }^{2}\right)}
$$

where $\mathrm{P}_{\mathrm{i}}$ : injection pressure $(\mathrm{Pa}), \mathrm{T}_{\mathrm{a}}$ : room temperature $(\mathrm{K}), \mathrm{P}_{0}=1.013 \cdot 10^{5} \mathrm{~Pa}, \mathrm{~T}_{0}=273 \mathrm{~K}$, $\mathrm{Q}_{\mathrm{i}}\left(\mathrm{T}_{0}, \mathrm{P}_{0}\right)$ : equivalent volumetric gas flow rate at normalized conditions $\left(\mathrm{m}^{3} / \mathrm{s}\right), \mu$ : nitrogen dynamic viscosity (Pas), L : specimen length $(\mathrm{m}), \mathrm{S}:$ specimen surface $\left(\mathrm{m}^{2}\right)$.

In this study, a COD sensor, used as a feedback signal of the tensile test, is placed in the aluminium compartment, between two points, determined by the position of the steel plates stuck on one flat face of a specimen, at mid-height of the latter and across the potential failure plane (see Fig. 1(b)). The two points are distanced by $10 \mathrm{~mm}$, sufficiently close to integrate only the area around the crack and sufficiently elongated to let the crack, not exactly rectilinear, pass through.

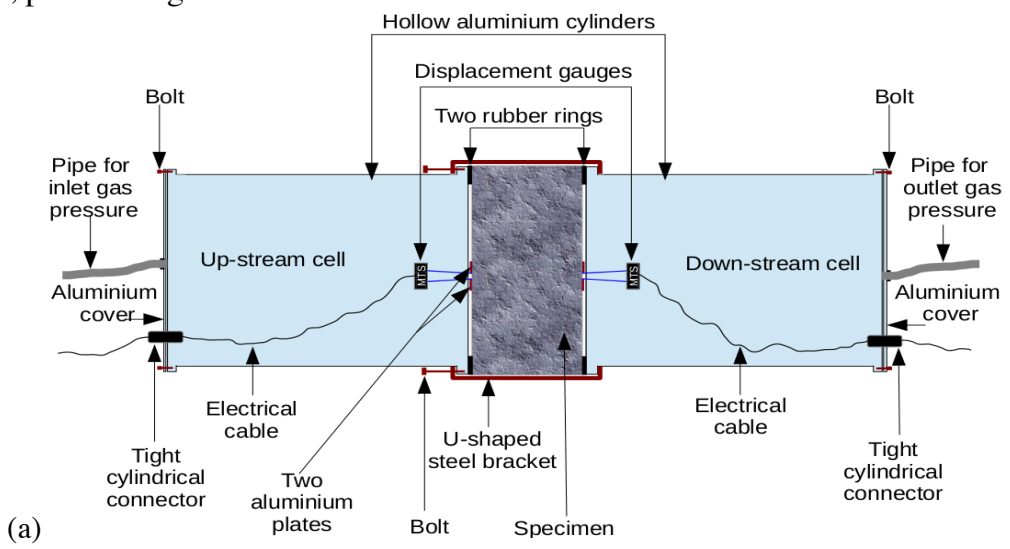

(b)
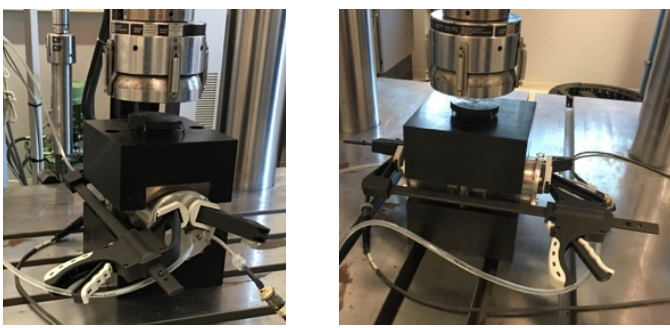

Fig. 2. (a) Side section view of the "double" loading-permeability device, (b) overall views of the "double" experimental device (GeM, Nantes University, IUT Saint-Nazaire).

The tests within the "simple" device, COD-controlled with a constant rate for loading and unloading, are performed within a hydraulic loading frame MTS $500 \mathrm{kN}$. A specimen, supplied with an aluminium compartment, is placed between two rigid metal platens of the loading frame equipped with plywood strips, in order to redistribute the load and prevent crushing at the loading points [2]. The second flat face of a specimen is left voluntarily free to allow the measurements of the displacement field by a Digital Image Correlation method, but the results won't be presented in this paper.

The second "double" device (see Fig. 2 (a)) includes two parts: one placed on the upstream side and the second one on the down-stream side, each one fitted with a COD sensor: 
COD 1 and COD 2. The principle is common to the first device, except the use of the incurved shape loading platens (see Fig. 2 (b)), instead of flat ones with plywood strips. Tightness and security elements are also modified: up-stream and down-stream cells are clamped in the perpendicular direction to the loading (see Fig. 2 (b)) and a $250 \mathrm{kN}$ MTS loading cell is employed within a hydraulic loading frame CFM / MTS $1000 \mathrm{kN}$. Gas pressure and flow rates are measured here at the up-stream and at the down-stream sides and the feedback signal of the tensile test corresponds to the mean COD value between COD 1 and COD 2 .

\subsubsection{Validation and Testing procedure}

The external diameter of the aluminium compartments is $10 \mathrm{~mm}$ lower than the diameter of a "disc" specimen in order to not interfere with the boundary conditions of the mechanical test. Once attached to a specimen using two steel clips, it doesn't impact the mechanical behaviour: global rigidity of a specimen is not impacted by the permeability device, even under gas pressure.

As a Brazilian splitting test is unstable in the post-peak phase [16], use of a COD sensor on the only one flat face specimen is highly risky. For this reason, before a test within the "simple" device, each specimen needs to be geometrically investigated and the larger diameter face needs to be chosen as the COD-driven to avoid loss of control. In fact, a slight difference of about $0.05-0.1 \mathrm{~mm}$ in diameter between two faces, due to mould imperfections, is sufficient to perform tests successfully, despite nonsymmetric crack initiation. On the other hand, as the injected gas pressure occurs some flexural effects and therefore may promote crack propagation on the non-driven face within the "simple" device, some tests may not succeed by loss of control. For this reason, results of four mortar specimens will be presented, instead of six initially desired.

As the "double" device permits to control the tests by a mean COD value, there is no risk of collapse: all the tests, realised on micoconcrete, two concretes and macroconcrete succeded.

Testing procedure: Before a test, a specimen, supplied with loading-permeability device(s), is positioned on the lower platen of the loading frame and alignment control is performed (force driven test) when the upper platen approaches a specimen to contact it. After this stage, the controlled variable is switched and mechanical loading becomes CODdriven ("simple" device) or mean COD-driven ("double" device) with a constant rate of $0,02 \mu \mathrm{m} / \mathrm{s}$. In order to avoid any specimen failure within the "simple" device, due to coupled mechanical and gas pressure effects, after each loading step, a specimen is slightly unloaded, and then gas pressure imposed and permeability test performed. This procedure is maintained until cracking propagates to the non-driven face. The latter occurs well after the peak load and demonstrates like a step in F-COD response (see Fig. 3 (a)). Once the step is exceeded, permeability tests may be performed under full loading, nevertheless accompanied by stress relaxation effects, shown in Fig. 3(a).

\section{Results and discussion}

The initial permeability of the mortar specimens varies between $9.8 \cdot 10^{-17} \mathrm{~m}^{2}$ and $12.3 \cdot 10^{-17}$ $\mathrm{m}^{2}$. This variation is relatively small and illustrates the good reproducibility of the material in each specimen.

The variation of gas permeability with the COD during loading within the "simple" device is plotted in Fig. 3(b). One may say that, for all the four mortar specimens tested, results fall on the same master sigmoid curve. The latter presents three characteristic phases, delayed to the mechanical ones: the first (1) one (up to COD of 30/40 microns) where the permeability 
increases relatively slightly, the second (2) one (COD of 30/40-80 microns) where the permeability increases rapidly and the third (3) one (COD beyond 80 microns) which is characterized by a slower rate than the latter (see Fig. 3(b)). The first phase corresponds to the gas transfer through both the mortar pores and the incompletely developed crack on the stronger diameter face, which propagates to the second face. The second phase corresponds probably to the gas flow by the crack "passing-through" with increasing opening and length, while the third phase (3) is probably related to the localized gas flow through the crack for which only the opening increases. These observations on sigmoid-like tendencies are in qualitative agreement with the numerical purposes of Pijaudier-Cabot et al. [17] as well as with the experimental results obtained by other researchers performing indirect tensilepermeability tests [3, 6-7] as well as compressive tests [13]. For the latter, permeability increase is more important around the peak-load in comparison to the Brazilian tensile test. This effect is probably due to numerous micro-cracks and their connectivity development in comparison to only one main crack formation and propagation in the Brazilian test. Indeed, recent modelling, carried out by the authors of this study [1], based upon a 3D lattice approach to represent the heterogeneity of the material and the failure mechanism, has highlighted good quantitative agreement between the numerical simulations and the experimental results for the range $[25 \mathrm{~mm} ; 125 \mathrm{~mm}]$. This comparison has to be seen as a starting point for a future coupled approach between numerical and experimental investigations and not as a complete validation considering the fact that only one realization has been regarded for the numerical simulations.
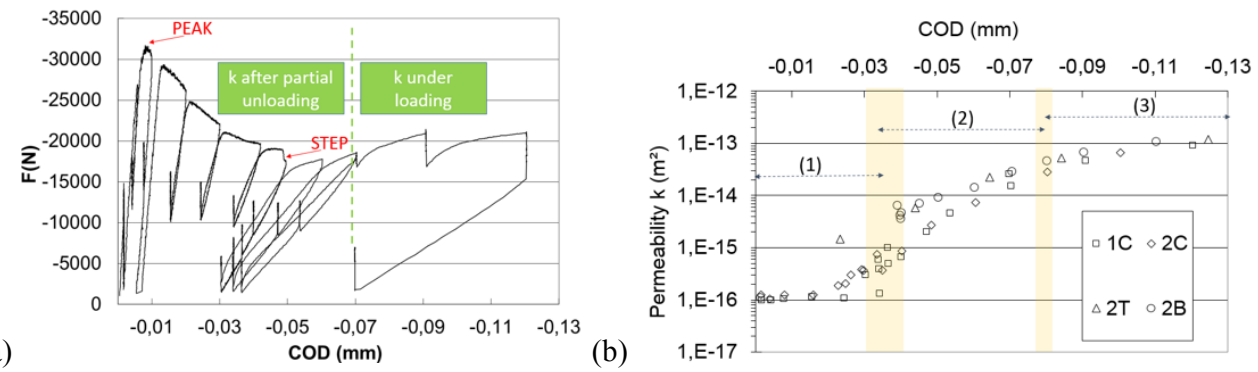

Fig. 3. (a) F-COD typical relation for a mortar specimen with two phases corresponding to different permeability test procedures, (b) variation of gas permeability with COD for four mortar specimens during tensile loading in the Brazilian splitting test.

Fig. 4(a) plots the experimental results obtained on microconrete, concrete and macroconcrete, within the "double" device. The tendencies concerning the sigmoidal evolutions, observed for mortar, are still confirmed. Here, permeabilities are reported to the permeability of the concrete (equal to $3.6 \cdot 10^{-17} \mathrm{~m}^{2}$ ). Moreover, one may observe that permeability increases not only with cracking, but also with aggregate size. These observations are in qualitative agreement with recent numerical simulations based on the already mentioned 3D lattice approach (see Fig. 4(b)). Besides, these results confirm other observations [13] concerning permeability increase with limestone aggregate size. By the way, permeability increase with aggregate size has also been recently confirmed for other materials, containing limestone sand and glass spheres as a model aggregate [18].

Fig. 5 plots permeability evolution for all the tested specimens and materials (with 2 different cements) within the "simple" and the "double" devices. One may observe that all the results fall on the same master curve, function of the permeability reported to the initial one of each material, and to the COD reported to the peak COD at the failure side. These results assume that a relative permeability evolution follows a single sigmoid law, independently to other parameters. Therefore, one may say that the overall evolution of the permeability may follow a multiplicative format: $\mathrm{k}=\mathrm{f}$ (tensile cracking) $* \mathrm{~g}$ (aggregate size). The increase of permeability 
is the product of two functions ( $\mathrm{f}$ and $\mathrm{g}$ ) which reflect each contribution separately. In other words, permeability increase due to tensile cracking seems to be decoupled from the aggregate size effect.

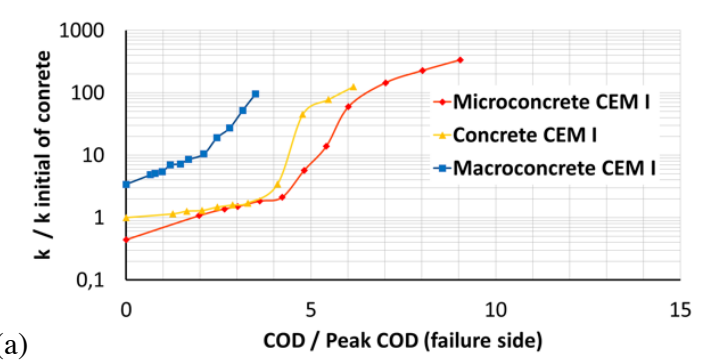

(b)

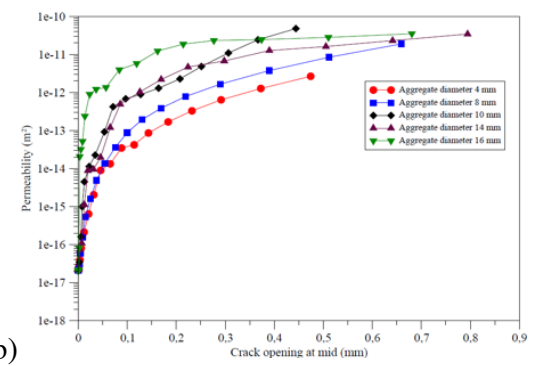

Fig. 4. (a) Relative permeability evolution with crack opening and aggregate size, (b) numerical permeability evolution with cracking and aggregate size (mono-size inclusions: 4,8,10,14,16mm)[18].

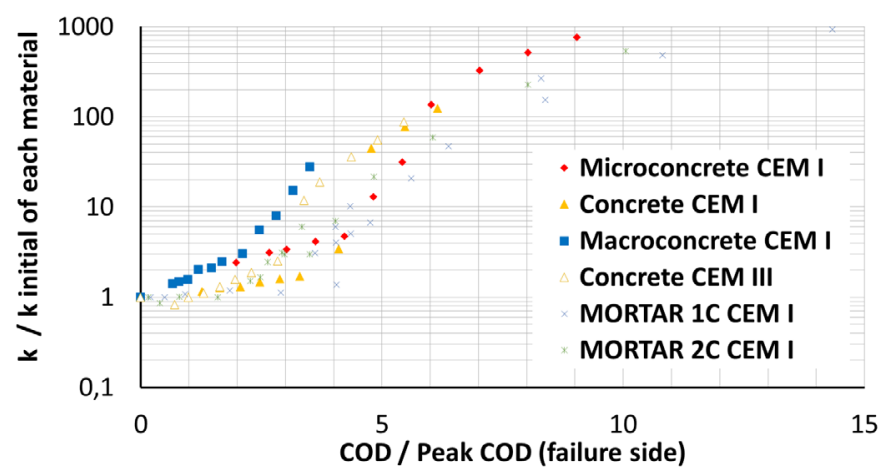

Fig. 5. Relative permeability evolution for all the tested materials and specimens within the "simple" and the "double" devices.

\section{Concluding Remarks}

The results reported in this study can be summarised as follows:

- The developed testing method has several advantages: cracking is produced by tensile stress and crack opening displacement is measured during loading simultaneously to gas permeability.

- Permeability increase depending on the crack opening (COD) follows a sigmoid curve, already observed for the compressive tests [14]. The experimental results are in quantitative agreement for the range $[25 \mu \mathrm{m} ; 125 \mu \mathrm{m}]$ with numerical simulations, based upon a 3D lattice approach to represent the heterogeneity, developed by the authors of this study [1].

- Three regimes with different rates may be distinguished for the sigmoidal evolution, depending on crack connectivity and geometry. The first one corresponds to a slight increase of permeability which starts beyond the peak load due to nonsymmetric crack initiation. Then, an important increase of permeability is observed, corresponding to the gas flow by the passing-through connected crack with increasing opening and length. The third phase with moderate rate is probably related to the localized gas flow through the crack for which only the opening increases. 
- Permeability increases not only with the COD, but also with the aggregate size, independently of their nature and shape (limestone crushed aggregates, glass spheres). The experimental results are in qualitative agreement with numerical simulation results based on the mesoscopic modelling $[1,18]$.

- Experimental results highlight that the overall evolution of a relative permeability depending on the COD follows a single sigmoid law for all the materials tested. The effects of cracking and of aggregate size may therefore be separated by two distinct functions. Further studies with different concretes and a wider range of aggregate size, nature and volume fractions are needed to expand this relationship.

\section{References}

1. N. Benkemoun, H. Al-Khazraji, P. Poullain, M. Choinska, A. Khelidj, Int. J. Numer. Anal. Methods Geomech. 1-20 (2017)

2. K. Wang, J. Daniel, S. Surendra, K. Alan, Cem Concr Res. 27(3), 381-93 (1997)

3. C.M. Aldea, S.P. Shah, A. Karr, Mater. Struct. 32(219), 370-76 (1999)

4. A. Djerbi, S. Bonnet, A. Khelidj, V. Baroghel-Bouny, Cem Concr Res. 38(6), 877-883 (2008)

5. V. Picandet, A. Khelidj, H. Bellegou, Cem. Concr. Res. 39(6), 537-547 (2009)

6. B. Gerard, Contribution des couplages mécanique-chimie-transfert dans la tenue à long terme des ouvrages de stockage de déchets radioactifs ( $\mathrm{PhD}$ Thesis, Ecole Normale Supérieure de Cachan, 1996)

7. G. Rastiello, C. Boulay, S. Dal Pont, J.L. Tailhan, P. Rossi, Cem. Concr. Res. 56, 20-28, (2014)

8. S. Granger, A. Loukili, G. Pijaudier-Cabot, G. Chanvillard, Cem. Concr. Res. 37(4), 519-527 (2007)

9. Z. A. Kameche, F. Ghomari, M. Choinska, A. Khelidj, Constr. Build. Mater. 65, 551565 (2014)

10. J. Bisschop, J. Van Mier, J. Mater. Struct. 35(8), 453-461 (2002)

11. P. Grassl, H.S. Wong, N.R. Buenfeld, Cem. Concr. Res. 40(1), 85-93 (2010)

12. A.M. Grabiec, D. Zawal, J. Szulc, Constr. Build. Mater. 98, 17-24 (2015)

13. A. Fabien, M. Choinska, S. Bonnet, A. Khelidj, Key Eng. Mater. 729, 115-121 (2017)

14. M. Choinska, A. Khelidj, G. Chatzigeorgiou, G. Pijaudier-Cabot, Cem. Concr. Res. 37, 79-88 (2007)

15. M. Choinska, F. Dufour, G. Pijaudier-Cabot, A. Huerta, A. Khelidj, How to extract a crack opening from a continuous damage finite element computation? Application for the estimation of permeability (ISTE - Wiley. GeoProc 2008, Lille, France, J.F. Shao \& N. Burlion Eds., 2008)

16. R. Gettu, B. Mobasher, S. Carmona, D. Jansen, Cem. Based Mater. 3(2), 54-71 (1996)

17. G. Pijaudier-Cabot, F. Dufour, M. Choinska, J. Eng. Mech. 135, 1022-1028 (2009)

18. H. Al-Khazraji, Influence of crack opening, aggregates size and volume fraction on hydro-mechanical properties of concrete in a Brazilian splitting test: $3 D$ meso-macro scale modeling and experimental work (PhD Thesis, Université de Nantes, 2017) 